\section{Clinical ocular pharmacology}

Jimmy D Bartlett and Siret D Jaanus

Butterworth-Heinemann, 4th

edition, 2001, £70 ISBN 0750670398

Eye (2002) 16, 681.

doi:10.1038/sj.eye.6700052

Bigger and brighter than the third edition this is a most welcome update to what is justifiably considered the gold standard in basic pharmacology of the eye.

This substantial book consisting of 38 chapters weighs 3 kilograms and has 48 contributing authors. Chapters are grouped into four parts, the fundamentals of ocular pharmacology; the pharmacology of ocular drugs; ocular drugs used in clinical practice and ocular toxicology.

Inclusion of the non steroidal anti-inflammatory drugs (NSAIDs) with selectivity for the cox-2 iso enzymes is most welcome in the chapter on analgesics as is some structured guidance in the first chapter where use of topical drugs in pregnancy looks at current FDA classification.

In the second part of this new edition a chapter on the pharmacological management of refractive surgery replaces a topic on contact lens care reflecting current trends within the speciality.

Glaucoma drugs are updated to include brinzolamide and a summary of the clinical advantages of topical carbonic anhydrase inhibitors (CAIs) whilst the prostaglandin section discusses the use of latanoprost in greater depth and includes the newer agents unoprostone and travoprost. The section on glaucoma management includes updated prescribing advice which excludes pilocarpine and focuses on beta blockers, brimonidine, carbonic anhydrase inhibitors or a combination of two or more of these. The classification of anti-infective agents now includes mechanisms of action and the chapter on the retina gives a brief explanation of photodynamic therapy with appropriate references.

There are significant useful additions to the chapter on ocular lubricants, in particular a chart classification of dry eye conditions plus a section on tear stimulation as a future therapeutic option. The chapter on allergic eye disease has been significantly improved by providing a clear introduction to immunology with the different types of allergic hypersensitivity reactions and has useful tables for ease of reference to both disease states and treatment options.

Section four on toxicology covers drug interactions and the data in this new edition are more succinct detailing only those of clinical significance. Ocular effects of systemic drugs include rifabutin associated uveitis and ocular changes with sildenafil and vigabatrin. In this edition there is an emphasis on the need to report any suspected adverse events although the mechanism for reporting is only relevant to the USA.

The final chapter on the management of systemic emergencies has been expanded to include drug-related reactions and offers defensive strategies to prevent or minimise adverse drug events. Each chapter is well supported by references with an emphasis on sources from peerreviewed literature.

The attractive style with bolder print has meant some compromises where text shown in tables has been abbreviated and where for this latest edition comparative figures have not been reproduced on adjacent pages.
Although the use of chemical structures may not be considered clinically relevant I would like to have seen a few more retained. Structures of antivirals in the previous edition, for example, usefully highlight structural similarities to target DNA nucleosides.

Some data, such as the chart of commonly used abbreviations is only relevant to American practice and indeed use of these in the UK could lead to dispensing errors. Another minor criticism is the occasional inconsistency with trade and generic drug nomenclature.

This invaluable book, with a wealth of reference material is relevant to all ophthalmic professionals, and well worth the price.

$$
\begin{array}{r}
\text { V Haylor } \\
\text { Ophthalmic Pharmacist } \\
\text { Royal Hallamshire Hospital } \\
\text { Sheffield, UK }
\end{array}
$$

\section{Binocular vision and orthoptics- investigation and management \\ B Evans and S Doshi \\ Butterworth-Heinemann, Oxford, 2001 \\ $£ 39.99$, ISBN 0750647132}

Eye (2002) 16, 681-682.

doi:10.1038/sj.eye. 6700185

This publication is a compilation of 16 articles that were published by various authors in the journal Optician between 1998 and 2000. The articles have been updated to form 16 chapters. There are 11 contributors, mainly optometrists but also an ophthalmologist and orthoptist.

Binocular Vision and Orthoptics is specifically aimed at eye-care 
professionals who encounter binocular vision anomalies in the primary care sector. It therefore concentrates on binocular vision conditions typically presenting to optometrists, such as heterophoria and convergence insufficiency. Investigation and management of comitant and incomitant strabismus, and chapters giving a basic summary of medical and surgical management of binocular vision anomalies, are also included. The final chapter consists of three detailed case studies. Multiple choice questions and detailed answers are provided for each chapter.

The book is clearly presented incorporating helpful diagrams and plentiful use of check lists and summary boxes. It provides a useful overview of binocular vision anomalies in a concise format, but would provide insufficient detail for those wishing to specialise in the subject.

By the nature of this book's origin, many topics are covered in more than one chapter leading to repetition of information. The editors acknowledge this by stating that presentation of different authors' approaches helps to give a balanced perspective. This is true in some instances, however repetition of information, such as $\mathrm{AC} / \mathrm{A}$ ratio formulae, clinical tests for determining near point of convergence, clinical investigation of suppression, retinal correspondence and stereoacuity, becomes a little tedious.

Chapter 2 describes microtropia as a deviation of between 1 to $10^{\circ}$ and not 1 to $10 \Delta$. This may be a typing error but could create confusion. Chapter 3 recommends elimination of suppression in strabismus if the motor deviation can be corrected. However, this is without emphasis on the caution needed to establish whether the patient has potential for stable binocular single vision prior to commencing this treatment. It does however recommend that clinicians with little experience of more complex aspects of binocular vision would be best avoiding this form of treatment, chapter 8 reiterates this point. In chapter 12, Hess chart plots are of disappointing quality; one is presented upside down and the example given for superior oblique palsy shows an exo deviation on down gaze, which is not typical. Chapter 15 discusses the surgical management of strabismus and begins with the structural organisation of the ocular motor system and preoperative assessment of the patient. This section includes a description and diagram of Bielschowsky's head-tilt test and shows head tilt to the right in a suspected left superior oblique palsy to observe elimination of the deviation. This however is not the correct method and would not be diagnostic. The final part of this chapter outlines the fine detail of the surgical procedure involved in recession and resection operations (eg type of hook, sutures and clamps used), this provides unnecessary detail for the intended reader however it may provide an insight to what happens in theatre.

Chapters 8 and 9 provide excellent résumés of theoretical and clinical aspects of suppression and abnormal retinal correspondence respectively. The use of Botulinum Toxin in strabismus is clearly summarised in Chapter 14.

In spite of some of the aspects outlined above, the book achieves its aim in providing a useful practical guide to investigation and treatment of binocular vision anomalies for practitioners working in primary care.

HJ Griffiths

Department Opthalmology $\mathcal{E}$ Orthoptics

\section{University of Sheffield \\ Royal Hallamshire Hospital \\ Sheffield, UK}

\section{Plastic and orbital surgery}

Richard Collin and Geoffrey Rose (Editors)

BMJ Books, London, 2001, £55.00, ISBN 072791457 8, 200 pp

Eye (2002) 16, 682-683. doi:10.1038/sj.eye.6700175

It is always a pleasure to receive a parcel from our esteemed Editor, particularly when it contains a book and on a topic of great personal interest. The book is a volume in the Fundamentals of Clinical Ophthalmology Series under the overall editorship of Professor Lightman. The stated target audience of the series, is that of Ophthalmologists in Training and General Ophthalmologists wishing to update their knowledge. The editors of this volume are Messrs Collin and Rose, recognised authorities in their respective fields of oculo-plastics and orbits. They further define the purpose of their book as being to provide a practical guide to the management of basic oculo-plastic problems. Seventeen authors, all of whom have undertaken at least part of their training in Moorfields, with the Editors, contribute chapters. These cover the range of relevant anatomy and oculo-plastic, orbital and lacrimal disorders. Each of the 17 chapters stands alone. The chapters are adequately illustrated. Some of the illustrations are annotated, most are not, some of the line drawings are downright confusing.

The book does fulfil the stated aims of the authors. It is a pity that neither of them have contributed chapters. One of the problems of the text is the lack of uniform style. One or two minor errors have crept in, for example, Traquair is not 